\title{
Light pollution around Tonantzintla Observatory
}

\section{José A. Vázquez-Mata ${ }^{1}$, Héctor M. Hernández-Toledo ${ }^{1}$, Luis A. Martínez-Vázquez ${ }^{1}$ and Atanacio Pani-Cielo ${ }^{2}$}

\author{
${ }^{1}$ Instituto de Astronomía, Universidad Nacional Autónoma de México \\ Apto. Postal 70-264, 04510, México, D.F., México \\ email: jvazquez@astro.unam.mx hector@astroscu.unam.mx lamb@astroscu.unam.mx \\ ${ }^{2}$ Observatorio Astronómico Nacional, Tonantzintla, \\ Luis Enrique Erro, Tonantzintla, Puebla 72840, México \\ email:apani@astroscu.unam.mx
}

\begin{abstract}
Being close to the cities of Puebla to east and Cholula to the north, both having potential for large growth, the National Astronomical Observatory in Tonantzintla (OANTonantzintla) faces the danger of deteriorating its sky conditions even more. In order to maintain competitiveness for education and scientific programs, it is important to preserve the sky brightness conditions. through: 1) our awareness of the night sky characteristics in continuous monitoring campaigns, doing more measurements over the next years to monitor changes and 2) encouraging local authorities about the need to regulate public lighting at the same time, showing them the benefits of such initiatives when well planed and correctly implemented.
\end{abstract}

Keywords. light pollution, observatories, sky brightness

\section{Introduction}

In 1958 Guillermo Haro being director of the Observatorio Astronómico Nacional (OAN) of the Universidad Nacional Autónoma de México (UNAM), did an effort to provide the observatory with a modern reflector telescope that would be installed on the town of Tonantzintla, Puebla. The 1-m telescope at OAN-Tonantzintla was opened to the astronomical community in 1961 and served as a major astronomical facility in México. Soon after 1969 this site became progressively inhabilitated for professional astronomy mainly due to the increasing light pollution from the growing city of Puebla. However, the close collaboration between Facultad de Ciencias and Instituto de Astronomía at UNAM quickly brought the 1-m telescope at OAN-Tonantzintla as one of the first professional telescopes in México to be made available at the college level providing physics and astronomy students real access to modern scientific instruments.

Light pollution has been identified as a major problem for the OAN-Tonantzintla. The integrity of the site is being ensured by implementing sky brightness monitoring campaigns that will lead us to propose, along with the local authorities, a lighting regulation program for the site. We present the results of a campaign to estimate the local sky brightness in the neighborhood of the observatory from a visual estimate of the apparent magnitude of some known stars. We present our procedure and briefly discuss the results obtained.

\section{The National Astronomical Observatory at Tonantzintla}

Tonantzintla is located near Cholula in the state of Puebla $150 \mathrm{~km}$ away from the Instituto de Astronomía headquarters in México City. The main telescope is a 1-m 
f/15 Cassegrain with an equatorial mount. Recently the local sky conditions at the OAN-Tonantzintla were re-evaluated and the capabilities/limitations of the available instrumentation have been reviewed for future astronomical and educational programs Hernández-Toledo et al. (2010). From those results, a program to update and install new photometric and spectroscopy instrumentation for educational purposes is in progress and hence the urgency of doing a parallel preservation program of the sky conditions at the site.

\subsection{Light pollution at $O A N$-Tonantzintla}

Light pollution at OAN-Tonantzintla arises mainly from scattering of light emitted by sodium and mercury vapor and incandescent street lamps from the city of Puebla and nearby villages. Figure 1 is a night-time exposure of the telescope building at the OANTonantzintla. At the background, it is possible to observe the light-pollution effect magnified by cloud reflections of the lighting of the nearby city of Puebla.

\subsection{Sky surface brightness}

We have estimated the sky surface brightness of the local sky into a $8 \mathrm{~km}^{2}$ area around the Observatory. This was achieved through the visual estimate of the apparent magnitude of a set of known bright stars in the Orion constellation. This work was carried out with the collaboration of students at the undergraduate and graduate programs in physics and astronomy at UNAM (Figure 2).

\subsection{Procedure}

(a) Since stars in the Orion constellation show a wide range of apparent magnitudes, we use the sky surface brightness calibration (in mag $\mathrm{arsec}^{-2}$ ) from the visual estimate of the apparent magnitude of stars up to the natural eye limit of 6 mag Schaefer (1990).

(b) Identification charts of the stars in the Orion constellation were prepared in 1-mag steps to avoid wrong magnitude estimates as much as possible.

(c) A set of locations to carry out these measures, free of trees and tall buildings, were chosen and their longitude and latitude coordinates were estimated.

(d) At each location the students made a visual estimate of the apparent magnitude of the observed stars toward the Orion constellation, identifying and matching as close as possible, the observed configuration of stars with that in the identification charts. After that, they moved $1 \mathrm{~km}$ away to proceed with a new measure.

\section{Results}

The left panel of Fig. 3 shows a view of OAN-Tonantzintla at a height of $6 \mathrm{~km}$ taken by Google Earth. "OAN-T" tag indicates the telescope local area and the rest of the points

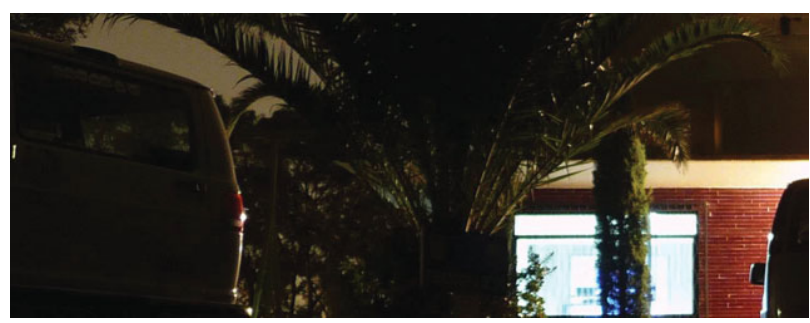

Figure 1. Image of the 1-m telescope building at the OAN-Tonantzintla. A light-pollution fuzz formed by the lighting of the nearby city of Puebla dominates the background. Photo by J.A. Vázquez-Mata. 


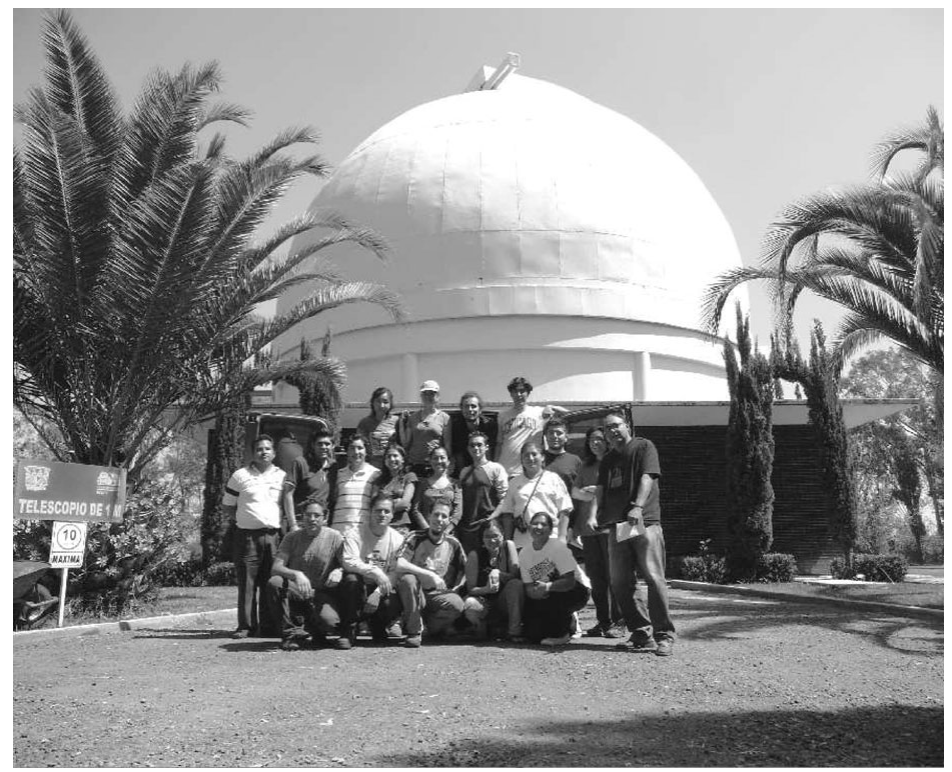

Figure 2. Students participating in the experiment.

show the area $\left(8 \mathrm{~km}^{2}\right)$ where the sky surface brightness measurements were carried out. The right panel shows a set of contours with representative values of the resulting sky surface brightness distribution after a $2 \mathrm{D}$ spline fitting function to the data.

The bright regions show the influence of the public lighting on our estimates. The dark regions at the bottom of the figure are interpreted as spurious effects due to the lack of data at those locations. A representative value of the sky surface brightness around the OAN-Tonantzintla (excluding the very local zone of the telescope) is $18.6 \mathrm{mag} \operatorname{arcsec}^{-2}$. As a comparison, a sky surface brightness value of $18.5 \mathrm{mag} \operatorname{arcsec}^{-2}$ from our CCD observations towards the city of Puebla was obtained. In accordance with this results,
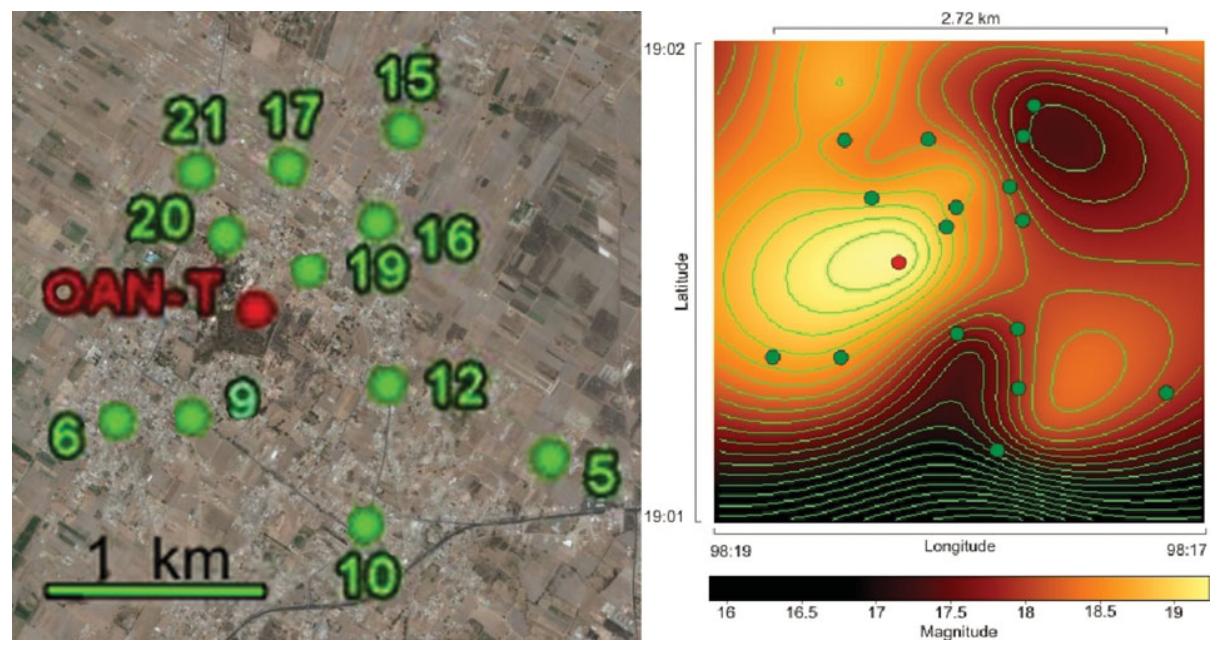

Figure 3. (Left) A view of the OAN-Tonantzintla area at a height of $6 \mathrm{~km}$, the red point indicates the location of OAN, and the green points are the sites of measurements. (Right) Surface brightness distribution of the sky around OAN-Tonantzintla (clear colors represent darker zones). 
in a similar but larger-scale experiment a reliability of the order of $0.65 \mathrm{mag} \operatorname{arcsec}^{-2}$ is expected under good weather conditions.

\section{Conclusion}

The brightness of the moonless night sky above OAN-Tonantzintla has been measured from the visual estimate of the apparent magnitude of bright stars in the Orion constellation. The median sky brightness in an area of about $8 \mathrm{~km}^{2}$ around the observatory is $V=$ $18.6 \mathrm{mag} \operatorname{arcsec}^{-2}$. In spite of the present sky conditions, it is still possible to do reasonable astronomical research but excellent educational work under well planned observing strategies and good weather conditions. A program to improve the shielding efficiency of public lighting must be implemented in the nearby villages surrounding Tonantzintla Observatory. Continued strong involvement of the observatory and the local authorities is needed to ensure our current major effort to update and transform this facility for education. Similar experiments can be implemented in sites where light pollution could start to be a problem. An example of a successful preservation program in México we mention the statement of a new law to preserve the dark sky around our major observatory at San Pedro Mártir in Baja California, México (see http://www.ordenjuridico. gob.mx/Estatal/BAJA\%20CALIFORNIA/Municipios/Ensenada/55REG.pdf).

\section{Acknowledgements}

JAVM and HMHT acknowledge the collaboration of the students: Aldo Rodríguez, Tula Bernal, Hugo Méndez, Pablo Miranda, Enrique Anzures, Rodrigo Sacahui, Leticia Rivera, Yax k'in Coronado, Roberto Figuera, Luis Avendaño, Federico Ortiz, Teresita Suárez, Alejandra Jiménez, Sergio Ortega, Rebeca Baños, Ivonne Medina, Santiago Torres, Abraham Avellana, Héctor Bustos, Carolina Castellanos, Jesús Zendejas, Carolina León \& Alan Miralrio. The authors acknowledge the municipal presidents of Santa María Tonantzintla, San Rafael Comac \& San Francisco Acatepec for their help during this campaign.

\section{References}

Schaefer, B. 1990, PASP, 102, 212

Hernández-Toledo, H.M. et al. 2010, RMxAA, 46, 47 